\section{Neurodermitistag der WAPA, Bochum 2004} Cave: Kontaktallergie auch
schon bei den ganz Kleinen!

\author{
Kontaktsensibilisierungen sind bei Kindern keineswegs selten. Bei \\ entsprechendem klinischen Bild kann eine Testung sogar bereits im \\ Säuglingsalter sinnvoll sein - besonders wenn ein atopisches \\ Ekzem vorliegt.
}

$\mathrm{D}$ ie Inzidenz des allergischen Kontaktekzems im frühen Kindesalter liegt bei etwa $1,5 \%$. Ab dem 8 . Lebensjahr ist ein stetiger Anstieg zu verzeichnen, bis im Alter von etwa 14 Jahren die gleiche Inzidenz wie bei Erwachsenen erreicht wird - je nach Literaturquelle $13-24 \%$.

\section{Bei Verdacht in jedem Alter testen}

Bei Neurodermitispatienten sei die Inzidenz des Kontaktekzems jedoch deutlich höher, erläuterte Dr. Marcus Freitag: Bis zu $43 \%$ aller Betroffenen leiden mindestens an einer Kontaktallergie, so der Bochumer Allergologe. Dies rechtfertige im Verdachtsfall eine Testung, völlig unabhängig vom Alter des Patienten. Die übliche Epikutantestung am Rücken ist dabei durchaus auch bei Kleinkindern bereits möglich. $\mathrm{Zu}$ beachten ist allerdings, dass im Kindesalter eher irritative Reaktionen auftreten

Häufige Kontaktallergene im
Kindesalter
— Metalle (Nickel, Kobalt, Kalium-
dichromat)
— Thiomersal
— Quecksilber
- Duftstoffe, Perubalsam
- Kolophonium
- Wollwachsalkohole
- Klebstoff- oder Gummi-Inhaltsstoffe
- Neomycin
- Bufexamac
— Dispersionsfarben (z. B. in Textilien)

können, die nicht als Allergie fehlgedeutet werden dürfen.

Ein Verdacht auf eine Kontaktallergie sei, so Freitag, bereits bei besonders hartnäckigen Krankheitsbildern gegeben: Es könnte sich eine Kontaktallergie gegen das angewendete topische Medikament dahinter verbergen. Einzelne Bestandteile der Basispflegemittel, die bei Neurodermitis eingesetzt werden, zählen mit zu den häufigsten Kontaktallergenen, beispielsweise Salbengrundlagen wie Wollwachsalkohole.

\section{Haarspray und Handy-Hüllen als Allergenquellen}

Bei Säuglingen und Kleinkindern sei zu beachten, so Freitag, dass eine Kontaktallergie auch gegen Allergene bestehen könne, mit denen das Kind selbst gar nicht direkt in Kontakt kommt, beispielsweise das Haarspray der Mutter. "Schuhdermatitiden" entstehen leicht bei eng anliegendem Schuhwerk. Mögliche Auslöser sind hier Gerbstoffe, Gummiadditiva oder Farbstoffe. Bei Schulkindern treten öfter Kontaktallergien gegen Bestandteile von Klebstoffen oder Radiergummi auf. Zu den häufigsten Auslösern von Kontaktallergien zählen Metalle (Nickel, Kobalt, Kaliumdichromat), die in Ohrschmuck, Zahnspangen, Piercings oder verchromten Handy-Hüllen enthalten sind. ic

Freitag M. Bedeutung von Kontaktsensibilisierungen bei der atopischen Dermatitis. 5. Neurodermitistag der Westdeutschen Arbeitsgemeinschaft für pädiatrische Allergologie (WAPA), Bochum, 15. Mai 2004

\section{Hautkrebs als Berufskrankheit}

Die Zahl der Basalzellkarzinome und der spinozellulären Karzinome sowie der malignen Melanome nimmt progredient zu. Beruflich stark UV-exponierte Menschen zeigen häufiger Präkanzerosen und Hautkrebs als die übrige Bevölkerung. Gleichwohl zählt der UV-induzierte Haut-

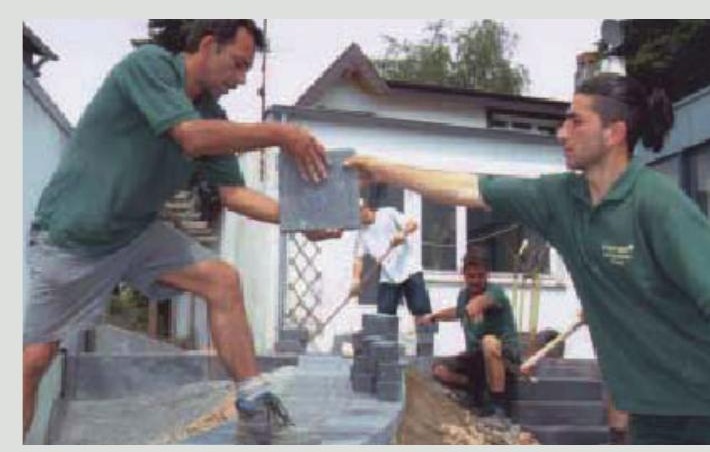

krebs nicht zu den Berufskrankheiten. Eine sozialrechtliche Anerkennung nach $\S 9$ Abs. 2 SGB VII ist allgemein an folgende Voraussetzungen gebunden:

1. Die Exposition einer bestimmten Personengruppe bei ihrer Arbeit muss erheblich höher sein als bei der übrigen Bevölkerung.

2. Nach medizinischem Wissensstand muss eine generelle Eignung dieser Einwirkung bestehen, Krankheiten dieser Art zu verursachen.

3. Die entsprechenden medizinischen Erkenntnisse waren bei der letzten Ergänzung der Anlage 1 zur Berufskrankheitenverordnung noch nicht ausreichend oder nicht geprüft worden.

Die generelle Eignung und eine erhöhte Inzidenz sind gegeben. Probleme bei einer Anerkennung von Hautkrebs als Berufskrankheit bereiten aber die Definition eines Grenzwertes der UV-Dosis und die Abgrenzung zwischen beruflicher und privater UV-Exposition. Im Allgemeinen wird von einer Risikoverdoppelung ausgegangen: Bei Überschreitung eines relativen Risikos von zwei im Vergleich zur Normalbevölkerung überwiegen die arbeitsbedingten Anteile als Auslöser. Eine solche Risikoverdoppelung ist für das spinozelluläre Karzinom bei ausschließlich UV-bedingter Hautkrebsentstehung errechnet worden. Insofern scheinen die Voraussetzungen für eine Anerkennung als Berufskrankheit zumindest für diese Tumorentität gegeben.

Wehrmann W. Beruflich bedingter Hautkrebs - von der Prävention zur Anerkennung. 7. Krefelder Hautschutztag, Krefeld, 22./23. Juni 2004 